\title{
Accuracy of robot-assisted versus conventional freehand pedicle screw placement in spine surgery: a systematic review and meta- analysis of randomized controlled trials
}

\author{
Yu-Ning Peng ${ }^{1 \#}$, Li-Cheng Tsai ${ }^{1 *}$, Horng-Chaung Hsu ${ }^{2}$, Chia-Hung Kao ${ }^{3,4,5,6}$ \\ ${ }^{1}$ Department of Medicine, ${ }^{2}$ Department of Orthopedic Surgery, China Medical University Hospital, Taichung; ${ }^{3}$ Graduate Institute of Biomedical \\ Sciences and School of Medicine, College of Medicine, China Medical University, Taichung; ${ }^{4}$ Department of Nuclear Medicine and PET Center, \\ China Medical University Hospital, Taichung; ${ }^{5}$ Department of Bioinformatics and Medical Engineering, Asia University, Taichung; ${ }^{6}$ Center of \\ Augmented Intelligence in Healthcare, China Medical University Hospital, Taichung \\ Contributions: (I) Conception and design: YN Peng, CH Kao; (II) Administrative support: CH Kao; (III) Provision of study materials or patients: All \\ authors; (IV) Collection and assembly of data: All authors; (V) Data analysis and interpretation: All authors; (VI) Manuscript writing: All authors; (VII) \\ Final approval of manuscript: All authors. \\ "These authors contributed equally to this work. \\ Correspondence to: Chia-Hung Kao, MD. Graduate Institute of Biomedical Sciences and School of Medicine, College of Medicine, China Medical \\ University, No. 2, Yuh-Der Road, Taichung 40447. Email: d10040@mail.cmuh.org.tw; dr.kaochiahung@gmail.com.
}

\begin{abstract}
This systematic review and meta-analysis investigated differences in accuracy, operation time, and radiation exposure time between robot-assisted and freehand techniques for pedicle screw insertion. Two investigators independently searched for articles on randomized controlled trials (RCTs) published from 2012 to 2019. The final meta-analysis included seven RCTs. We compared the accuracy of pedicle screw placement, operation time, and radiation exposure time between robot-assisted and conventional freehand groups. Seven RCTs included 540 patients and placement of 2,476 pedicle screws, of which 1,220 were inserted using the robot-assisted technique and 1,256 were inserted using the conventional freehand technique. The pedicle screw positions were classified using the Gertzbein and Robbins classification (grade A-E). The combined results of Grade A [odds ratio $(\mathrm{OR})=1.68 ; 95 \%$ confidence intervals (CI): 0.82-3.44; $\mathrm{P}=0.16)$, Grade $\mathrm{A}+\mathrm{B}(\mathrm{OR}=1.70 ; 95 \% \mathrm{CI}: 0.47-6.13 ; \mathrm{P}=0.42)$, and Grade $\mathrm{C}+\mathrm{D}+\mathrm{E}(\mathrm{OR}=0.59 ; 95 \% \mathrm{CI}$ : $0.16-2.12 ; \mathrm{P}=0.42$ ) for the accuracy rate revealed no significant difference between the two groups. Subgroup analysis results revealed that the TiRobot-assisted technique presented a significantly improved pedicle screw insertion accuracy rate compared with that of the conventional freehand technique, based on Grade A, Grade A+B, and Grade C+D+E classifications. The SpineAssist-assisted technique presented an inferior pedicle screw insertion accuracy rate compared with that of the conventional freehand technique, based on Grade A, Grade A+B, and Grade C+D+E classifications. No difference between the Renaissance-assisted and conventional freehand techniques was noted for pedicle screw insertion accuracy rates, based on both Grade A (OR =1.58; 95\% CI: 0.85-2.96; P=0.15), Grade A+B (OR =2.20; 95\% CI: 0.39-12.43; P=0.37), and Grade $\mathrm{C}+\mathrm{D}+\mathrm{E}(\mathrm{OR}=0.45 ; 95 \% \mathrm{CI}: 0.08-2.56$; $\mathrm{P}=0.37)$ classifications. Regarding operation time, robotassisted surgery had significantly longer operation time than conventional freehand surgery. The robotassisted group had significantly shorter radiation exposure time. Regarding the pedicle screw insertion accuracy rate, the TiRobot-assisted technique was superior, the SpineAssist-assisted technique was inferior, and Renaissance was similar to the conventional freehand technique.
\end{abstract}

Keywords: Robot-assisted; radiation exposure; randomized controlled trials (RCTs); TiRobot-assisted technique; SpineAssist-assisted technique

Submitted Jan 30, 2020. Accepted for publication Jun 12, 2020.

doi: 10.21037/atm-20-1106

View this article at: http://dx.doi.org/10.21037/atm-20-1106

(c) Annals of Translational Medicine. All rights reserved. 


\section{Introduction}

Pedicle screw placement was first reported by Boucher in 1959 (1). Since then, pedicle screw insertion has gained acceptance by orthopedic surgeons and neurosurgeons and has been one of the most commonly used procedures in spine surgery over decades (2). To prevent injury to adjacent blood vessels and neural structures, accuracy in screw placement is essential. Navigation and imaging systems have been developed to improve the accuracy and safety of pedicle screw insertion (3); however, accurate placement still requires mastering surgical techniques and experience. Because of the presence of complex anatomical structures adjacent to the spine and wide variations between patients, accuracy of screw placement is challenging $(4,5)$. Pedicle screws are conventionally inserted without guidance by relying on anatomic landmarks, with or without the use of fluoroscopic navigation (6).

Recently, robot systems have been introduced to assist pedicle screw insertion. In total, 159 types of surgical robot prototypes were invented between 1998 and 2005 (7). High-accuracy robot-assisted spine systems have been developed and used to treat patients in many countries $(8,9)$. These systems were considered an improvement of spinal navigation that could increase the accuracy of screw placement and reduce intraoperative radiation exposure (8). Whether robot-assisted or conventional freehand technique has satisfactory outcome is controversial. To date, six systematic reviews and meta-analyses (10-14) comparing robot-assisted and conventional freehand pedicle screw placement have been published. However, only one study was a randomized controlled trial (RCT) (13). Fan et al. (14) reported that the robot-assisted technique is superior to the conventional freehand technique in terms of the accuracy rate of pedicle screw insertion. Staartjes et al. (11) concluded that robotic guidance has the potential to minimize the incidence of relevant postoperative revisions due to screw malposition. Liu et al. (12) and Gao et al. (13) have both reported an equivalent accuracy rate of pedicle screw insertion in both the techniques. Yu et al. (10) reported that the current literature cannot prove that the robot-assisted technique supersedes the freehand technique. Furthermore, $\mathrm{Li}$ et al. (15) reported that robot-assisted technique is more accurate in pedicle screw placement than the freehand technique. Therefore, it is necessary to perform an updated systemic review and meta-analysis. In this study, we performed a systematic review and meta-analysis based on RCTs to investigate differences in accuracy, operation time, and radiation exposure time between robot-assisted and conventional freehand pedicle screw placement techniques. We present the following article in accordance with the PRISMA reporting checklist (available at http://dx.doi. org/10.21037/atm-20-1106).

\section{Methods}

We performed this systemic review and meta-analysis based on the Preferred Reporting Items for Systematic Reviews and Meta-Analysis statement (16) and the recommendations of the Cochrane Handbook for Systematic Reviews of Intervention (17). Institutional review board approval was not necessary because this study reviewed previously published RCTs that did not involve any processing of individual patient data.

\section{Systematic search for trials}

We searched for RCTs in the National Library of Medicine of PubMed database, Web of Science, and Cochrane Library. MeSH terms and keywords used for the search in various combinations were robotic, robot-assisted, freehand, pedicle screws, bone screws, spine surgery, and spinal surgery. RCTs included in our review were published between April 15, 2012, and February 8, 2019. The reference lists of studies were also reviewed to search for additional studies, and the bibliographies of relevant systematic reviews were manually searched.

\section{Inclusion criteria}

Initially, two authors (YNP and LCT) independently screened and extracted data by applying the same standard criteria, and then, full articles were examined independently to determine whether they met our inclusion criteria. Discrepancies were resolved through discussion until a consensus was reached. We limited our search to RCTs performed on humans and published in English.

Studies were selected based on the following inclusion criteria:

(I) RCTs that compared robot-assisted and conventional freehand pedicle screw placement techniques in patients undergoing spinal surgery;

(II) RCTs assessing the accuracy of the pedicle screw placement by postoperative computed tomography (CT) scans;

(III) RCTs with sufficient data that would enable to 


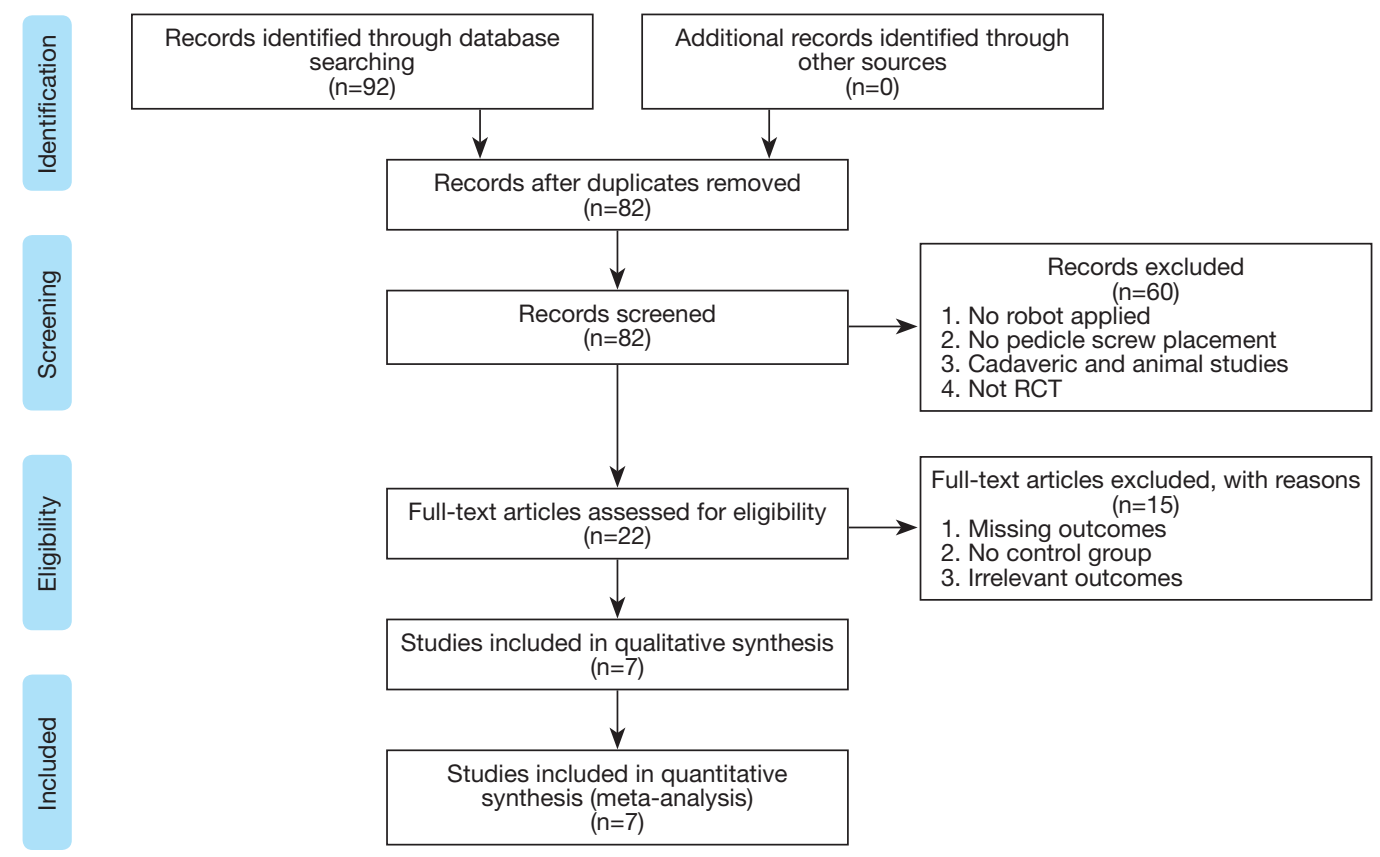

Figure 1 PRISMA flow chart depicting literature search and selection process.

perform meaningful comparison ( $>20$ pedicle screw placements surgeries in each study group).

Exclusion criteria were as follows:

(I) RCTs without a control group;

(II) Cadaveric and animal studies;

(III) Screw placement accuracy was not evaluated.

\section{Risk of bias assessment}

Two authors (YNP and LCT) independently evaluated each study based on the Cochrane risk of bias assessment scale (17). Seven categories of bias were evaluated: random selection, allocation concealment, blinding of participants and outcome assessment, outcome data, reporting bias, and other study bias. Three levels (low-, unclear-, and high-risk) were summarized in each category. Discrepancies in scoring were discussed among the authors.

\section{Data extraction}

Two authors (YNP and LCT) independently reviewed and extracted the following information from each study: name of the first author, country of origin, publication year, study type, anatomical level considered, types of robot used, surgery indication, sample size, types of surgery performed, number of patients, number of screws inserted, Grade A accuracy rate, Grade A+B accuracy rate, Grade $\mathrm{C}+\mathrm{D}+\mathrm{E}$ accuracy rate, operation time, and amount of radiation exposure. All data were extracted from tables or texts.

\section{Data analysis}

We conducted the meta-analysis using Review Manager 5.3 (Nordic Cochrane Centre, Cochrane Collaboration, Copenhagen, Sweden). Odds ratios (ORs) and weighted mean differences were used to compare continuous and dichotomous variables, respectively. All results are reported with $95 \%$ confidence intervals $(\mathrm{CI})$, and $\mathrm{P}<0.05$ was set as the level of significance. We used the Higgins $\mathrm{I}^{2}$ statistic to assess the heterogeneity of individual studies. A fixed-effects model was used if no obvious heterogeneity existed (i.e., $\mathrm{I}^{2}<50 \%$ ); otherwise, a random-effects model was used (if $\mathrm{I}^{2}>50 \%$ and $\left.\mathrm{P}<0.10\right)$.

\section{Results}

\section{Literature search}

The literature selection process is presented in Figure 1. The initial literature search yielded 92 potentially eligible studies from PubMed, Web of Science, and Cochrane Library, of which 10 studies were removed 
due to duplication. Furthermore, remaining 82 studies were screened for titles and abstracts and 60 studies were removed due to the following reasons: no robot applied, no pedicle screw placement performed, not an RCT, or a cadaveric and animal study. Full texts of 22 studies that had potential for inclusion were reviewed. Subsequently, 15 studies were removed due to missing outcomes, no control group, or irrelevant outcomes. Finally, 7 RCTs (1824) were deemed eligible and included in this systematic review and meta-analysis.

\section{Main characteristics of included studies}

In total, 7 RCTs were eligible for inclusion in this metaanalysis, comprising 540 patients with 2,476 screws inserted. Pedicle screws inserted by robotic assistance and conventional freehand techniques were 1,220 and 1,256, respectively. These studies were published between 2012 and 2019. The characteristics and results of patients in these trials are listed in Table 1 . Three studies $(18,22,23)$ were conducted in South Korea, two studies $(20,21)$ were conducted in Germany, and two studies $(19,24)$ were conducted in China. Regarding the robot system, two studies $(20,21)$ used SpineAssist (Mazor Robotics Ltd., Caesarea, Israel), three studies $(18,22,23)$ used Renaissance (Mazor Robotics Ltd., Caesarea, Israel), and two studies $(19,24)$ used TiRobot (TINAVI Medical Technologies Co., Ltd., Beijing, China).

\section{Quality of studies}

Figures 2,3 present the risk of all types of bias. All studies claimed randomization, but two studies $(20,21)$ did not report the method of random sequence generation. Five studies $(18,19,22-24)$ mentioned the details of random sequence generation, and five studies $(18,19,22-24)$ reported allocation concealment. Five studies (19,21-24) reported blinding of outcome assessors, but no studies reported blinding of participants and personnel. Thus, none of the eight studies were double-blinded.

\section{Meta-analysis results}

\section{Pedicle screw insertion accuracy (Grade A)}

Figure 4 presents the comparison of pedicle screw insertion accuracy according to the Gertzbein-Robbins Classification Grade A $(0 \mathrm{~mm})$. The random-effect model was used because heterogeneity between included trials was significant $\left(\mathrm{I}^{2}=77 \%, \mathrm{P}=0.0002\right)$. The combined results revealed that no significant difference existed between robot-assisted and conventional freehand techniques $\left(\tau^{2}=0.60, \chi^{2}=26.17, \mathrm{df}=6 ;\right.$ OR $=1.68,95 \%$ CI: $\left.0.82-3.44\right)$ based on the Grade A accuracy for pedicle screw placement.

We further performed a subgroup analysis considering the type of robot system used. The results of the subgroup analysis indicated that a statistically significant subgroup effect $\left(\mathrm{I}^{2}=92.0 \%, \mathrm{P}<0.00001\right)$ existed, meaning the type of the robot system significantly modified the level of accuracy achieved by the robot-assisted technique compared with the conventional freehand technique. The results of the subgroup analysis revealed that the TiRobot-assisted technique presented significantly improved accuracy (Grade A) for pedicle screw insertion compared with the conventional freehand technique $(\mathrm{OR}=3.22 ; 95 \% \mathrm{CI}$ : 2.07-5.01; $\mathrm{P}<0.00001)$. By contrast, the SpineAssist-assisted technique had inferior pedicle screw insertion accuracy (Grade A) compared with the conventional freehand technique (OR $=0.63 ; 95 \%$ CI: $0.39-1.00 ; \mathrm{P}=0.05$ ), whereas no difference was observed in the accuracy achieved by the Renaissanceassisted and conventional freehand techniques for pedicle screw insertion $(\mathrm{OR}=1.58 ; 95 \% \mathrm{CI}$ : 0.85-2.96; $\mathrm{P}=0.15)$. Although overall heterogeneity was high $\left(\mathrm{I}^{2}=77 \%, \mathrm{P}=0.0002\right)$, no heterogeneity was observed among studies that used robot systems, namely SpineAssist $\left(\mathrm{I}^{2}=0 \%, \mathrm{P}=0.45\right)$, Renaissance $\left(\mathrm{I}^{2}=0 \%, \mathrm{P}=0.79\right)$, and TiRobot $\left(\mathrm{I}^{2}=0 \%, \mathrm{P}=0.86\right)$.

\section{Pedicle screw insertion accuracy (Grade A+B)}

Figure 5 presents the comparison of pedicle screw insertion accuracy based on the Gertzbein-Robbins Classification Grade $\mathrm{A}+\mathrm{B}(0$ and $<2 \mathrm{~mm})$. The random-effect model was used because heterogeneity between included trials was significant $\left(\mathrm{I}^{2}=71 \%, \mathrm{P}=0.002\right)$. The combined results revealed that no significant difference existed between robot-assisted and conventional freehand techniques $\left(\tau^{2}=1.62, \chi^{2}=20.62, \mathrm{df}=6 ; \mathrm{OR}=1.70,95 \%\right.$ CI: 0.47-6.13) based on the Grade A+B accuracy for pedicle screw placement. Furthermore, the subgroup analysis was conducted based on the type of robot system used. The results of the subgroup analysis suggested that a statistically significant subgroup effect $\left(\mathrm{I}^{2}=89.8 \%, \mathrm{P}<0.0001\right)$ existed, meaning the type of the robot system significantly modified the accuracy achieved by the robot-assisted technique in comparison with the conventional freehand technique. The results of the subgroup analysis revealed that the TiRobotassisted technique presented significantly improved pedicle screw insertion accuracy (Grade $\mathrm{A}+\mathrm{B})$ compared with the 


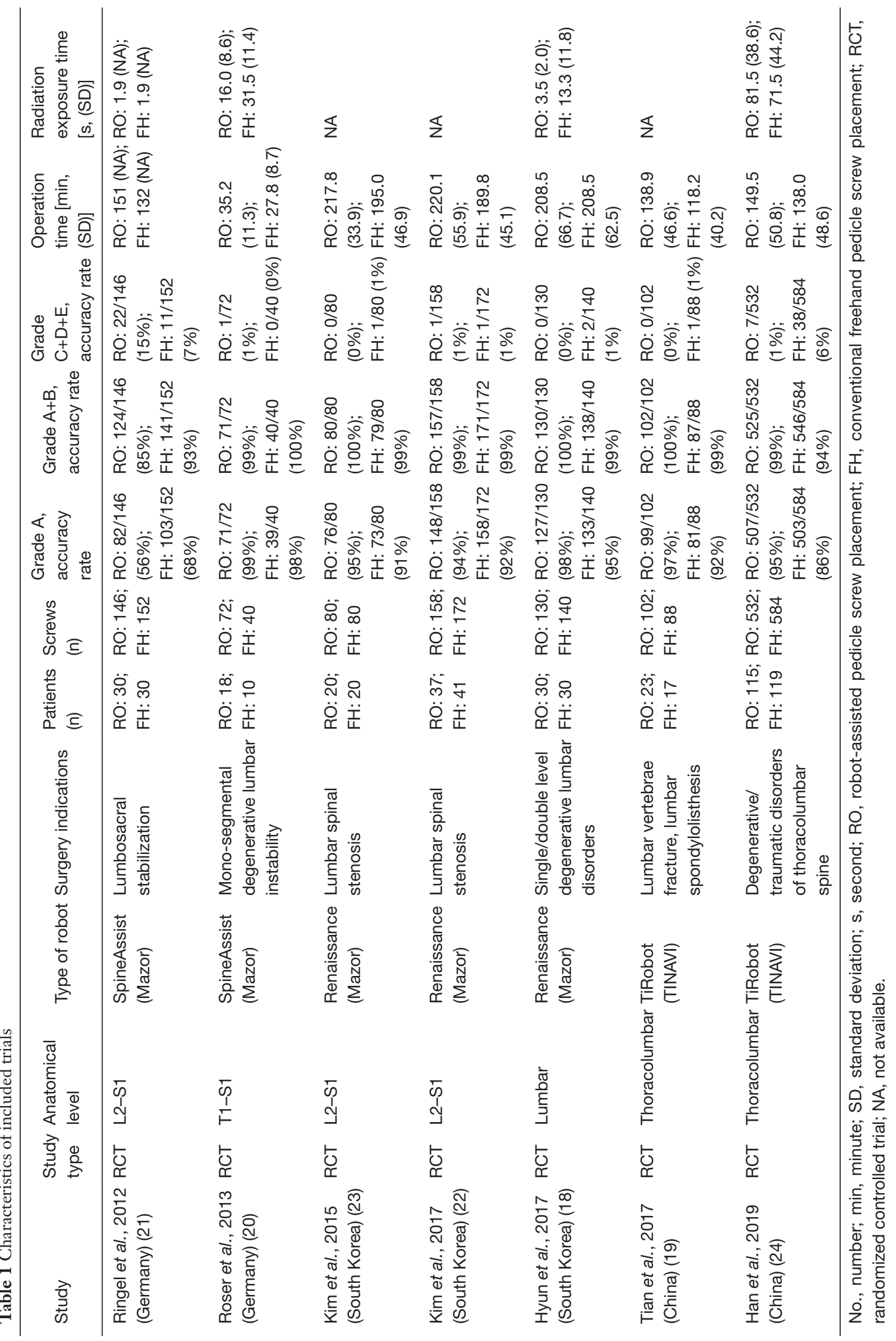




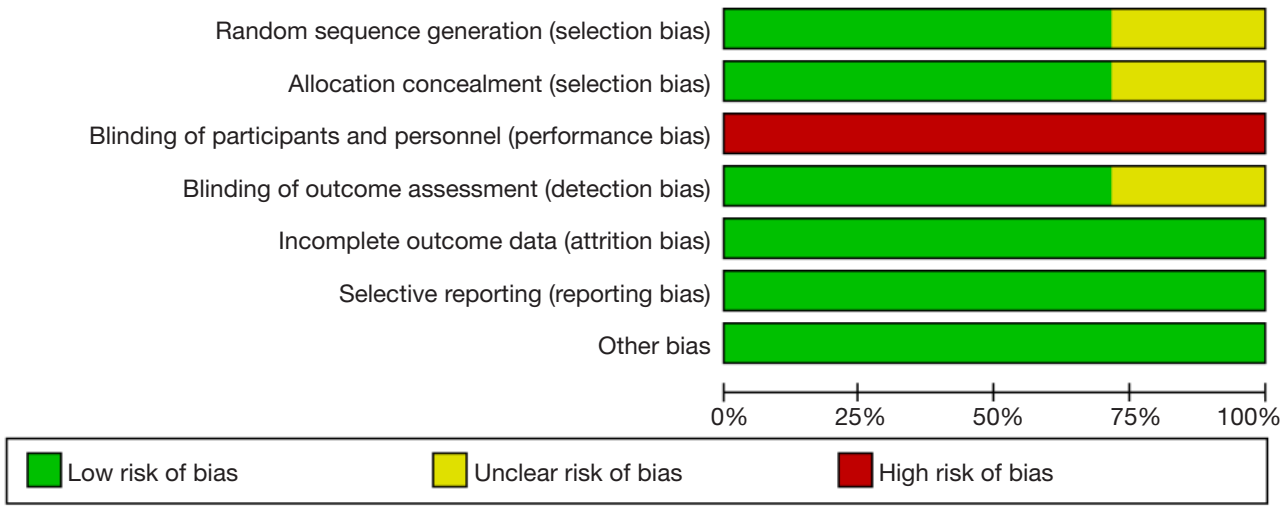

Figure 2 Risk of bias graph.

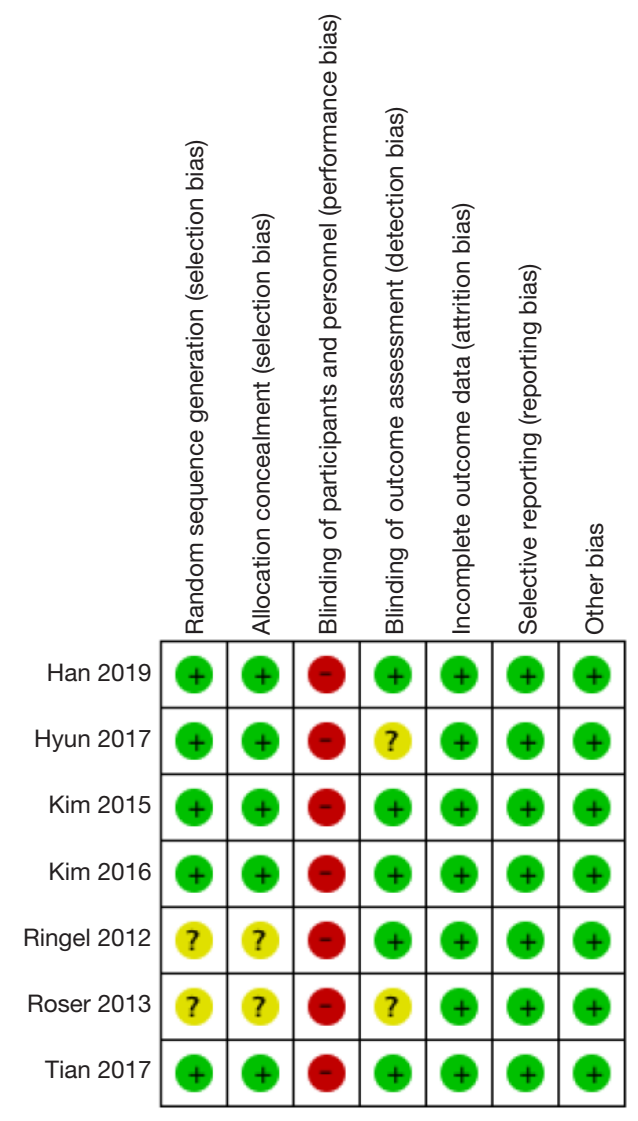

Figure 3 Risk of bias summary.

conventional freehand technique $(\mathrm{OR}=5.10 ; 95 \% \mathrm{CI}$ : 2.31-11.23; $\mathrm{P}<0.0001)$. By contrast, SpineAssist-assisted technique had inferior pedicle screw insertion accuracy (Grade $\mathrm{A}+\mathrm{B}$ ) compared with the conventional freehand technique $(\mathrm{OR}=0.5 ; 95 \% \mathrm{CI}: 0.21-0.94 ; \mathrm{P}=0.03)$, and no difference was observed for pedicle screw insertion between the Renaissance-assisted and conventional freehand techniques $(\mathrm{OR}=2.20 ; 95 \% \mathrm{CI}$ : $0.39-12.43 ; \mathrm{P}=0.37)$. Although overall heterogeneity was high $\left(\mathrm{I}^{2}=71 \%, \mathrm{P}=0.002\right)$, no heterogeneity was observed among studies using the robot systems, namely SpineAssist $\left(\mathrm{I}^{2}=0 \%, \mathrm{P}=0.86\right)$, Renaissance $\left(\mathrm{I}^{2}=0 \%, \mathrm{P}=0.72\right)$, and TiRobot $\left(\mathrm{I}^{2}=0 \%, \mathrm{P}=0.81\right)$.

\section{Pedicle screw insertion accuracy (Grade C+D+E)}

Figure 6 presents the comparison of pedicle screw insertion accuracy based on the Gertzbein-Robbins Classification Grade $\mathrm{C}+\mathrm{D}+\mathrm{E}$ (C: pedicle cortical breach $\geq 2$ to $<4 \mathrm{~mm}$ ); D: pedicle cortical breach $\geq 4$ to $<6 \mathrm{~mm}$; E: pedicle cortical breach $\geq 6 \mathrm{~mm}$ ), indicating inferior accuracy or even screw misplacement. The random-effect model was used because heterogeneity between included trials was significant $\left(\mathrm{I}^{2}=71 \%, \mathrm{P}=0.002\right)$. The combined results revealed that no significant difference existed between robot-assisted and conventional freehand techniques $\left(\tau^{2}=1.62, \chi^{2}=20.62, \mathrm{df}=6\right.$; $\mathrm{OR}=0.59 ; 95 \%$ CI: $0.16-2.12$ ) based on the Grade $\mathrm{C}+\mathrm{D}+\mathrm{E}$ accuracy for pedicle screw placement. Therefore, we further performed a subgroup analysis considering the type of robot system used. The results of the subgroup analysis revealed that a statistically significant subgroup effect $\left(\mathrm{I}^{2}=89.8 \%\right.$, $\mathrm{P}<0.0001)$ existed, meaning the type of the robot system significantly modified the accuracy achieved by the robotassisted technique in comparison with the conventional freehand technique. The results of the subgroup analysis revealed that the conventional freehand technique presented significantly inferior pedicle screw insertion accuracy (Grade $\mathrm{C}+\mathrm{D}+\mathrm{E})$ compared with the TiRobot-assisted technique (OR $=0.20$; 95\% CI: $0.09-0.43 \mathrm{P}<0.0001)$. By contrast, SpineAssist-assisted technique had inferior pedicle screw 


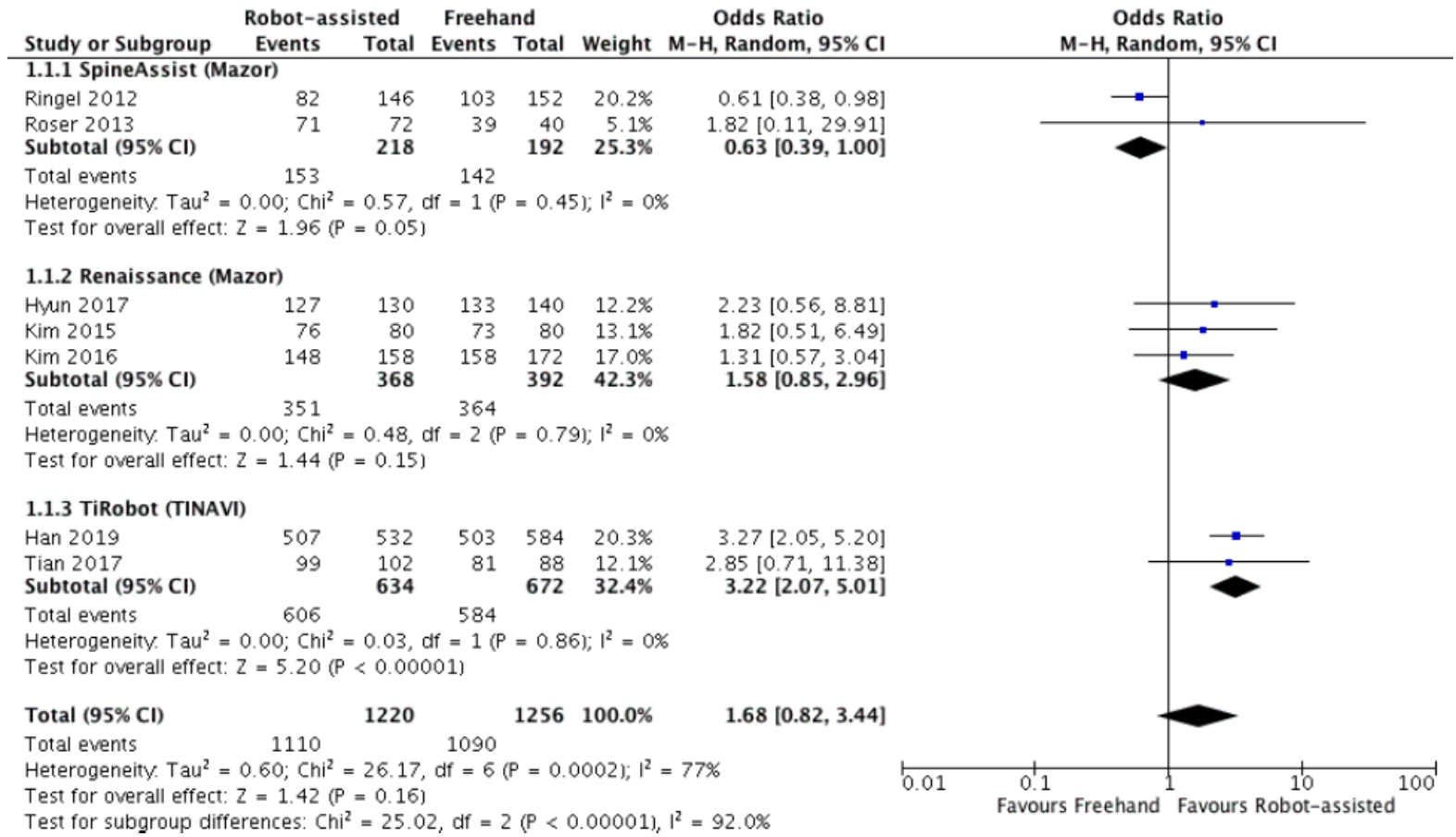

Figure 4 Pedicle screw insertion accuracy (Grade A): robot-assisted technique versus freehand technique.

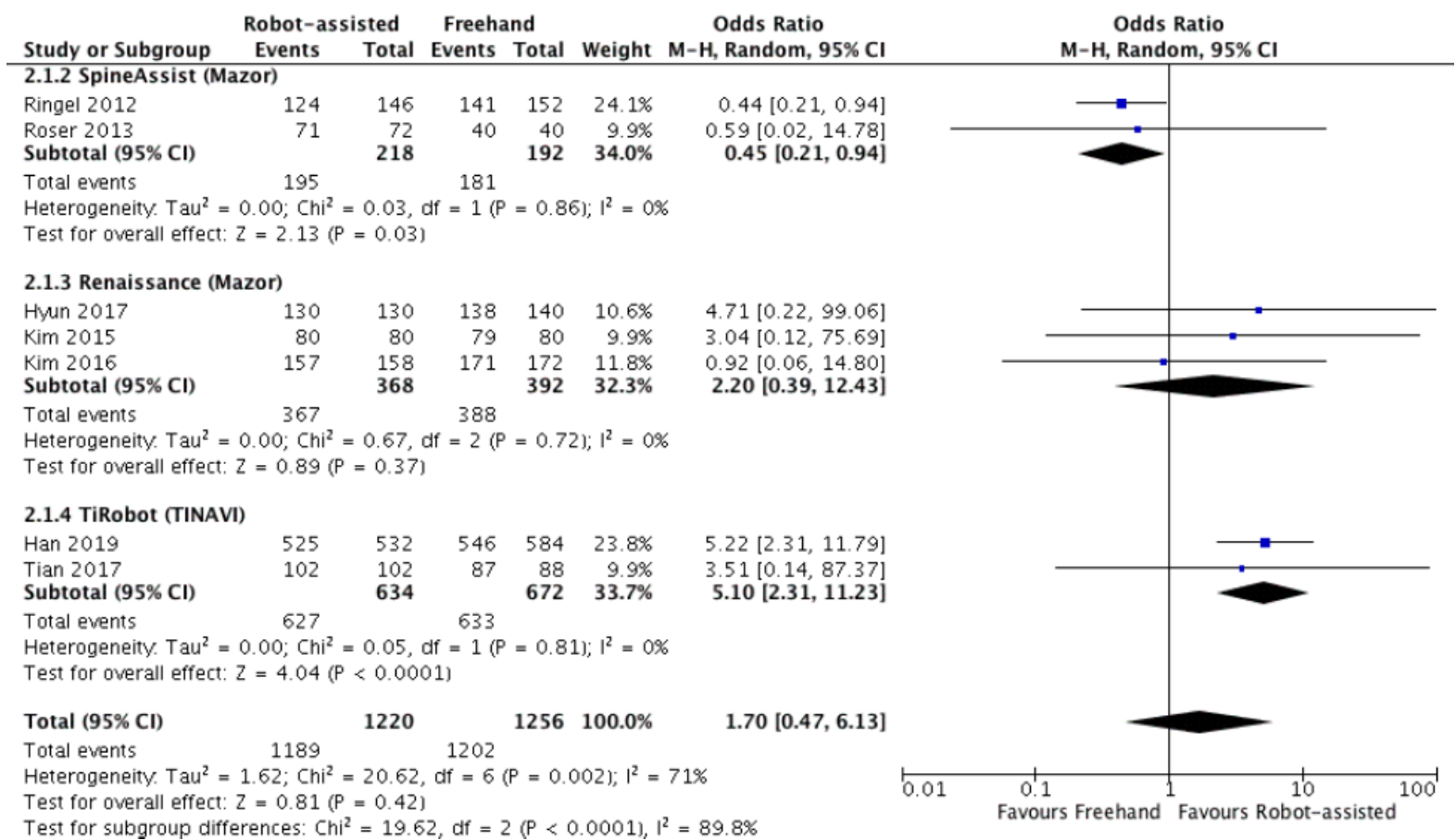

Figure 5 The comparison of pedicle screw insertion accuracy based on the Gertzbein-Robbins Classification Grade A+B (0 and $<2$ mm). 


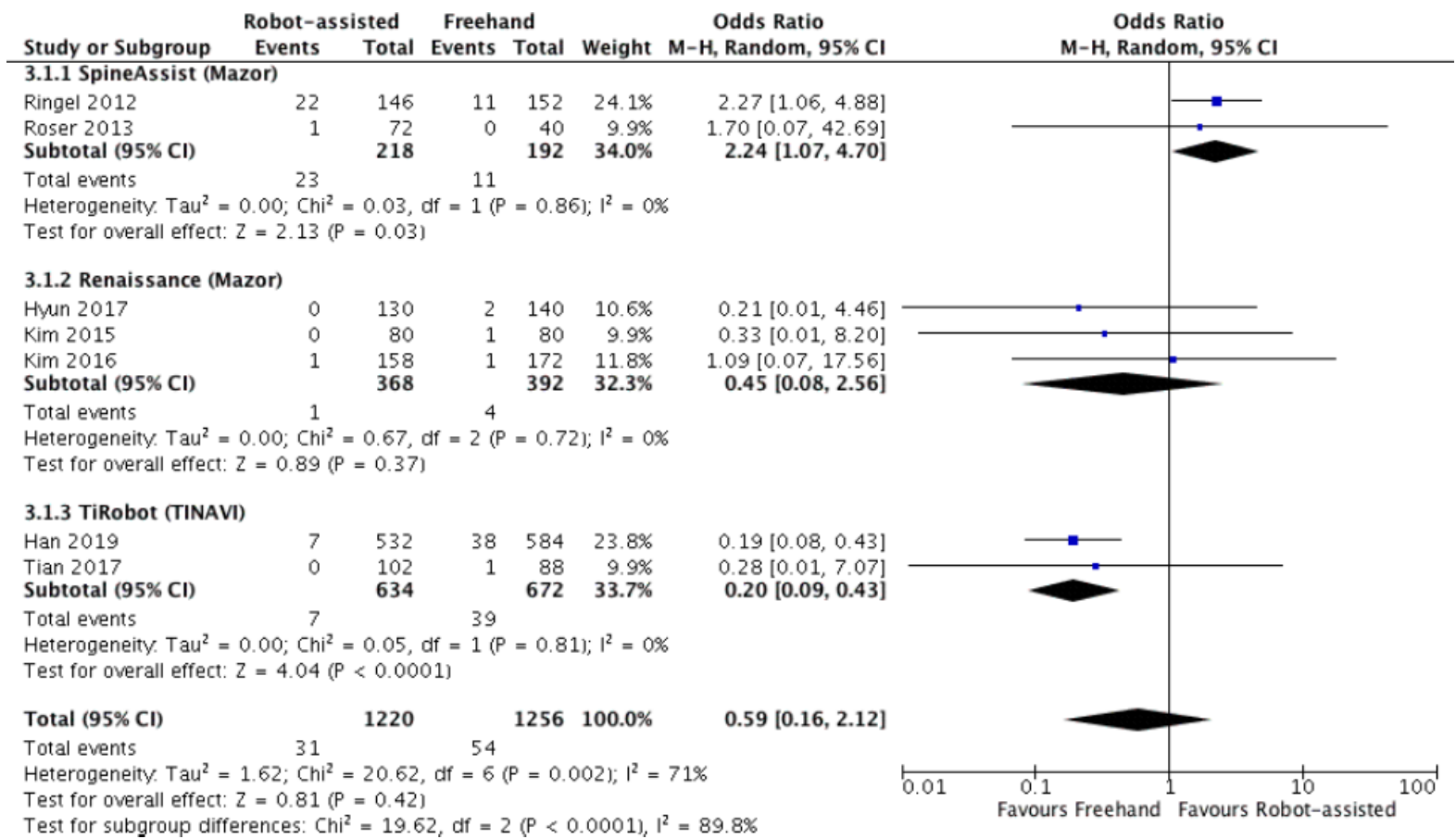

Figure 6 The comparison of pedicle screw insertion accuracy based on the Gertzbein-Robbins Classification Grade C+D+E.

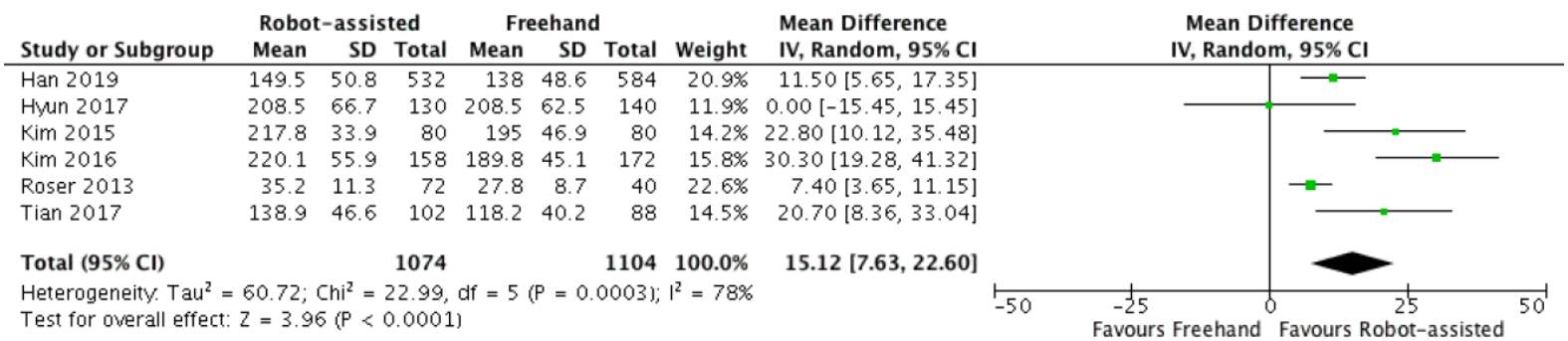

Figure 7 Operation time (min): robot-assisted versus freehand.

insertion accuracy (Grade $\mathrm{C}+\mathrm{D}+\mathrm{E}$ ) compared with the conventional freehand technique $(\mathrm{OR}=2.24 ; 95 \% \mathrm{CI}$ : 1.07-4.70; $\mathrm{P}=0.03$ ), and no difference was observed for pedicle screw insertion between the Renaissance-assisted and conventional freehand techniques ( $\mathrm{OR}=0.45 ; 95 \% \mathrm{CI}$ : $0.08-2.56 ; \mathrm{P}=0.37)$. Although overall heterogeneity was high $\left(\mathrm{I}^{2}=71 \%, \mathrm{P}=0.002\right)$, no heterogeneity was observed among studies using the robot systems, namely SpineAssist $\left(\mathrm{I}^{2}=0 \%, \mathrm{P}=0.86\right)$, Renaissance $\left(\mathrm{I}^{2}=0 \%, \mathrm{P}=0.72\right)$, and TiRobot $\left(\mathrm{I}^{2}=0 \%, \mathrm{P}=0.81\right)$.

\section{Operation time}

The meta-analysis of operation time is presented in Figure 7.
Six RCTs (18-20,22-24) mentioned the overall operation time in minutes. The random-effect model was used because the heterogeneity of results was high $\left(\mathrm{I}^{2}=78 \% ; \mathrm{P}=0.0003\right)$. Overall, the robot-assisted group had longer operation time compared with the conventional freehand group (MD, 15.12 min; 95\% CI: 7.63-22.60 min; $\mathrm{P}<0.0001$ ).

\section{Radiation exposure time}

Figure 8 presents the meta-analysis of radiation exposure time. Two RCTs $(18,20,24)$ mentioned the radiation exposure time per screw in seconds. The heterogeneity of results was high $\left(\mathrm{I}^{2}=84 \% ; \mathrm{P}=0.01\right)$; hence, the random-effect model was used. The meta-analysis revealed that the robot- 


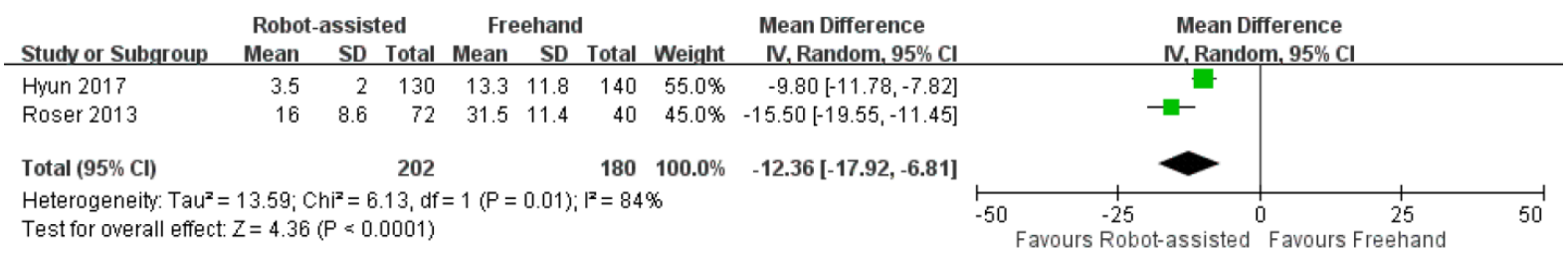

Figure 8 Radiation exposure time (s): robot-assisted versus freehand.

assisted group had significantly shorter radiation exposure time compared with the conventional freehand group (MD, -12.36 s; $95 \%$ CI: -17.92 to -6.81 s; $\mathrm{P}<0.0001)$.

Before the robot-assisted surgery, the preoperative CT scan covering the region of interest was reconstructed to form 3-dimensional images using the proprietary software. In both group of Hyun et al. (18), intraoperative radiography was performed using a biplane C-arm fluoroscope (Siremobil ISOC; Siemens, Erlangen, Germany) to assess the screw location. Regarding Roser et al. (20), two fluoroscopy (anteroposterior plane and $60^{\circ}$ oblique to the lateral plane) were taken intraoperatively when a fiducial array was placed on the mounting platform in the SpineAssist-assisted surgery, whereas using anteroposterior and lateral fluoroscopy (BV Endura; Philips, Eindhoven, The Netherlands) in conventional free-hand group.

\section{Discussion}

According to our knowledge and literature search, this is the systematic review and meta-analysis comparing the robotassisted and conventional freehand techniques for pedicle screw insertion based on RCTs and subgroup analysis based on the type of the robot system used. Six previously published meta-analyses revealed the following: one (16.7\%) study (11) concluded that the robot-assisted technique can minimize the incidence of relevant postoperative revisions caused by screw malpositioning. One (16.7\%) study (14) reported that the robot-assisted technique is superior to the conventional freehand technique. Two $(33.3 \%)$ studies $(12,13)$ reported that the robot-assisted and freehand techniques have an equivalent accuracy rate for screw insertion. One (16.7\%) study (10) concluded that the robot-assisted technique did not supersede the conventional freehand technique in the accuracy rate for pedicle screw insertion. One (16.7\%) study (18) reported that robot-assisted technique is more accurate in pedicle screw placement than the freehand technique. Among these studies, only Gao et al. (13) included RCTs as the included trials. Other studies $(10-12,14)$ included both RCTs and cohort studies for meta-analysis, which may have caused less powerful statistical results. In this study, eight RCTs were included for meta-analysis. All selected studies defined the accuracy of pedicle screw insertion by GertzbeinRobbins Classification (25). The combined results revealed no significant difference between the robot-assisted and conventional freehand techniques for pedicle screw insertion accuracy using Grade A, Grade A+B, and Grade $\mathrm{C}+\mathrm{D}+\mathrm{E}$ classifications. However, the clinical significance of misplaced pedicle screw in asymptomatic postoperative patient still remains unclear. Current evidence showed that not every misplaced pedicle screw results in complications or revision surgery. A high rate of screw misplacement does not correlate with neurological complication (26), this may be due to normal spinal anatomy contains an epidural "cushion" in spinal canal, which provides a margin for error of screw malposition.

Due to the high heterogeneity of combined results, we conducted a subgroup analysis based on robot systems. The results of the subgroup analysis revealed that the type of robot systems significantly modified the effect of the robotassisted technique in comparison with the conventional freehand technique based on Grade A ( $\mathrm{I}^{2}=92.0 \%$, $\mathrm{P}<0.00001)$, Grade $\mathrm{A}+\mathrm{B}\left(\mathrm{I}^{2}=89.8 \%, \mathrm{P}<0.0001\right)$, and Grade $\mathrm{C}+\mathrm{D}+\mathrm{E}\left(\mathrm{I}^{2}=89.8 \%, \mathrm{P}<0.0001\right)$ classifications. The findings of the subgroup analysis revealed that TiRobot significantly improved pedicle screw insertion accuracy compared with that of the conventional freehand technique in Grade A, Grade $\mathrm{A}+\mathrm{B}$, and Grade $\mathrm{C}+\mathrm{D}+\mathrm{E}$ classifications. By contrast, SpineAssist reduced the pedicle screw insertion accuracy (Grade A, Grade A+B, and Grade $\mathrm{C}+\mathrm{D}+\mathrm{E}$ ) compared with the conventional freehand technique. No difference in the pedicle screw insertion accuracy (Grade A, Grade A+B, and Grade $\mathrm{C}+\mathrm{D}+\mathrm{E}$ ) was observed between Renaissanceassisted and conventional freehand techniques. SpineAssist is a robot mounted on the spine, which can increase the accuracy rate of screw positioning and reduce intraoperative radiation exposure. $(27,28)$ Previous studies have revealed 
that it has been mostly used for lumbar pedicle screw insertion, with high accuracy (27-29). Renaissance is another type of miniature spine-mounted robot, and numerous studies have supported its merits $(8,9,18,20,23)$. These systems comprise miniature spine-mounted robot and a controlling work station. Surgeons preoperatively determine the position and trajectories based on CT scans. This robot is firmly connected to the spine of patients during the operation. Fluoroscopic images are captured and matched with preoperative CT scans during the surgery, and the robot provides the trajectory and entry point for the instrumentation. Surgeons can now accurately drill or instrument the target vertebra, and this process is repeated until all vertebrae are located and instrumented $(18,20)$. TiRobot was the first orthopedic surgical robot developed in China, which has a robotic arm with tracking abilities and is combined with an intraoperative $3 \mathrm{D}$ navigation system. Few studies have supported that TiRobot guidance can be feasible, safe, and accurate in spine surgery (30-32). During operation, fluoroscopic images are captured and automatically imported into the controlling workstation. Surgeons can plan the surgical trajectory for screw placement, including optimal position and dimensions of the implants in the axial, coronal, and sagittal views, and generate positioning orders for the robot arm. The arm automatically locates and moves according to orders from the controlling workstation and complete the surgical trajectory. After planning trajectories, a guide holder mounted on the robot arm spontaneously moves to the exact entry point according to the plan. Surgeon can drill guide pins and place screws through the holder $(24,30)$.

Operation time was measured in $6(86 \%)$ of our included trials (18-20,22-24,30). This meta-analysis found that robot-assisted surgery had significantly longer operation time compared with conventional freehand surgery. Ringel et al. (21), Tian et al. (19), Kim et al. (22,23), and Han et al. (24) reported longer operation times for robot-assisted surgery. By contrast, Hyun et al. (18) reported no difference between robot-assisted and conventional freehand surgeries. Methods specific to the robot-assisted technique may increase additional challenges for surgeons and increase operative time. Devices used to accurately guide pedicle screw insertion may also consume time.

Radiation exposure to the surgeon and patient during operation can be significant, especially in those patients who have abnormal anatomy structures and anatomic landmarks. Limiting radiation exposure during operation is also vital because excess amount of radiation can increase the malignancy risk (33). In our meta-analysis, two (25\%) RCTs $(18,20,24)$ have reported radiation exposure time per screw in seconds. Compared with conventional freehand surgery, Robot-assisted surgery was associated with significantly shorter radiation exposure time. Hyun et al. (18) reported that the fluoroscopy time per screw in conventional freehand surgery was nearly four times than that in robotassisted surgery. Roser et al. (20) found that radiation exposure time in conventional freehand surgery was nearly two times than that in robot-assisted surgery. With regard to the radiation dosage, Hyun et al. (18) found that Renaissance-assisted surgery significantly reduced between half and three quarters of the fluoroscopy dose compared with conventional freehand surgery, with the unit expressed in $\mathrm{mSv}$. Roser et al. (20) also reported that the radiation dosage was less in SpineAssist-assisted surgery, with the unit expressed in mGy. We excluded Han et al. (24) in terms of radiation exposure time, because they evaluated the radiation exposure time during the whole procedure, leading to the mean cumulative radiation exposure time relatively higher compared with the other studies. However, they found that overall radiation exposure time in conventional freehand surgery is not significantly more than that in robot-assisted surgery. In contrast, the cumulative radiation dosage is significantly less in robot-assisted surgery. It is possible to reduce the reliance on intraoperative fluoroscopy by using the robot-assisted technique because the surgeon left the operation room during the $3 \mathrm{D}$ imaging and C-arm can be removed after the preoperation plan, thus limiting radiation exposure to the surgeon. This study included only few trials and thus had high heterogeneity results $\left(\mathrm{I}^{2}=84 \%\right.$; $\mathrm{P}=0.01$ ). Therefore, adding more high-quality studies can strengthen the statistical efficacy of these findings. However, generally speaking, radiation exposure time is relevant with clinical experience of surgeons. Radiation exposure may be decreased with growing experience of surgeons, and most of our included RCTs were singlecenter studies including surgeons in different levels of training. The clinical experience of the surgeons was not investigated. Malik et al. (34) conducted a systemic review examining the association between clinical experience of surgeon and radiation exposure in orthopedic surgery. 18 studies evaluating radiation exposure in orthopedic surgery were included in the systemic review, and most of the studies showed that inexperienced surgeons, such as residents and fellows, had a higher radiation exposure and a higher total fluoroscopy time as compared to experienced surgeons. Thus, further study should include RCTs that 
discussed about the clinical experience of surgeons, in order to conduct a more thorough meta-analysis.

The robotic spine surgery systems have considerable additional costs, therefore, the cost-effectiveness of those techniques should take into consideration. Menger et al. (35) investigated the cost-effectiveness of robotic technology in spine surgery. This study concluded robotic surgery is a cost-effective technology, which is measured by rate of revision surgery, post-operative infection rate, length of stay, and operative time. Although robotic spine surgery systems are more expensive than conventional spine surgery systems, the rate of postoperative complications is less, which lead to lower total cost of hospitalization. During a 1-year period, robotic technology resulted in savings estimate of $\$ 608,546$ at a hospital which performed 557 elective spine surgeries (35).

Our study has some limitations. First, few RCTs were included. More RCTs with a larger sample size should be included. Only three studies used Renaissance, and each two studies used SpineAssist and TiRobot. Our findings revealed that different robot systems lead to significantly different results; thus, more studies should be included in each subgroup to achieve higher statistical efficacy. Second, most RCTs were conducted with small sample sizes.

The largest trial (24) included 234 patients, and the smallest trial (20) included 28 patients. Most of the trials included less than 100 patients. The results of treatment may be incorrect in small trials. Third, the heterogeneity of included studies was significant. This may be because patients in these studies had different surgical indications and anatomical levels and were treated with different types of robot. Although our meta-analysis had these mentioned limitations, it represents the highest level of evidence.

In conclusion, our meta-analysis suggested that the TiRobot-assisted technique was superior to the conventional freehand technique and Renaissance-assisted technique in terms of pedicle screw insertion accuracy. By contrast, the SpineAssist-assisted technique had a less pedicle screw insertion accuracy compared with the conventional freehand technique, and the Renaissance presented no difference between the two groups. Recent meta-analysis reported by $\mathrm{Li}$ et al. (15) also concluded that TiRobot-assisted technique is more accurate in pedicle screw placement than the Renaissance-assisted technique. The robot-assisted technique was associated with longer operation time and significantly shorter radiation exposure time. Robot-assisted spine surgery has the potential to improve the accuracy of pedicle screw insertion and may be a useful tool for surgeons. However, more RCTs with rigorous design should be included to further investigate the beneficial role of robotics in spine surgery.

\section{Acknowledgments}

Funding: This study is supported in part by Taiwan Ministry of Health and Welfare Clinical Trial Center (MOHW109TDU-B-212-114004), China Medical University Hospital (CMU107-ASIA-19); MOST Clinical Trial Consortium for Stroke (MOST 108-2321-B-039-003-), Tseng-Lien Lin Foundation, Taichung. The funders had no role in the study design, data collection and analysis, the decision to publish, or preparation of the manuscript. No additional external funding was received for this study.

\section{Footnote}

Reporting Checklist: The authors have completed the PRISMA reporting checklist. Available at http://dx.doi. org/10.21037/atm-20-1106

Conflicts of Interest: All authors have completed the ICMJE uniform disclosure form (available at http://dx.doi. org/10.21037/atm-20-1106). The authors have no conflicts of interest to declare.

Ethical Statement: The authors are accountable for all aspects of the work in ensuring that questions related to the accuracy or integrity of any part of the work are appropriately investigated and resolved.

Open Access Statement: This is an Open Access article distributed in accordance with the Creative Commons Attribution-NonCommercial-NoDerivs 4.0 International License (CC BY-NC-ND 4.0), which permits the noncommercial replication and distribution of the article with the strict proviso that no changes or edits are made and the original work is properly cited (including links to both the formal publication through the relevant DOI and the license). See: https://creativecommons.org/licenses/by-nc-nd/4.0/.

\section{References}

1. Boucher HH. A method of spinal fusion. J Bone Joint Surg Br 1959;41-B:248-59.

2. Malhotra D, Kalb S, Rodriguez-Martinez N. Instrumentation of the posterior thoracolumbar spine: from 
wires to pedicle screws. Neurosurgery 2014;10:497-504.

3. Youkilis AS, Quint DJ, McGillicuddy JE, et al. Stereotactic navigation for placement of pedicle screws in the thoracic spine. Neurosurgery 2001;48:771-8.

4. Tan SH, Teo EC, Chua HC. Quantitative threedimensional anatomy of cervical, thoracic and lumbar vertebrae of Chinese Singaporeans. Eur Spine J 2004;13:137-46.

5. Panjabi MM, Duranceau J, Goel V, et al. Cervical human vertebrae. Quantitative three-dimensional anatomy of the middle and lower regions. Spine (Phila Pa 1976) 1991;16:861-9.

6. Roy-Camille R, Saillant G, Mazel C. Plating of thoracic, thoracolumbar, and lumbar injuries with pedicle screw plates. Orthop Clin North Am 1986;17:147-59.

7. Pott PP, Scharf HP, Schwarz MLR. Today's state of the art in surgical robotics. Comput Aided Surg 2005;10:101-32.

8. van Dijk JD, van den Ende RPJ, Stramigioli S, et al. Clinical pedicle screw accuracy and deviation from planning in robot-guided spine surgery: robot-guided pedicle screw accuracy. Spine 2015;40:E986-91.

9. Devito DP, Kaplan L, Dietl R. Clinical acceptance and accuracy assessment of spinal implants guided with SpineAssist surgical robot: retrospective study. Spine (Phila Pa 1976) 2010;35:2109-15.

10. Yu L, Chen X, Margalit A, et al. Robot-assisted vs freehand pedicle screw fixation in spine surgery - a systematic review and a meta-analysis of comparative studies. Int J Med Robot 2018;14:e1892.

11. Staartjes VE, Klukowska AM, Schröder ML. Pedicle Screw Revision in Robot-Guided, Navigated, and Freehand Thoracolumbar Instrumentation: A Systematic Review and Meta-Analysis. World Neurosurg 2018;116:433-43.e8.

12. Liu H, Chen W, Wang Z, et al. Comparison of the accuracy between robot-assisted and conventional freehand pedicle screw placement: a systematic review and meta-analysis. Int J Comput Assist Radiol Surg 2016;11:2273-81.

13. Gao S, Lv Z, Fang H. Robot-assisted and conventional freehand pedicle screw placement: a systematic review and meta-analysis of randomized controlled trials. Eur Spine J 2018;27:920-30. http://dx.doi.org/10.1007/s00586-0175333-y

14. Fan Y, Du JP, Liu JJ, et al. Accuracy of pedicle screw placement comparing robot-assisted technology and the free-hand with fluoroscopy-guided method in spine surgery: An updated meta-analysis. Medicine (Baltimore) 2018;97:e10970.
15. Li HM, Zhang RJ, Shen CL. Accuracy of Pedicle Screw Placement and Clinical Outcomes of Robot-assisted Technique Versus Conventional Freehand Technique in Spine Surgery From Nine Randomized Controlled Trials: A Meta-analysis. Spine (Phila Pa 1976) 2020;45:E111-9.

16. Liberati A, Altman DG, Tetzlaff J, et al. The PRISMA statement for reporting systematic reviews and metaanalyses of studies that evaluate health care interventions: explanation and elaboration. PLoS Med 2009;6:e1000100.

17. Higgins JP, Green S. Cochrane Collaboration. Cochrane Handbook for Systematic Reviews of Interventions. Chichester, England; Hoboken, NJ: Wiley-Blackwell, 2008.

18. Hyun SJ, Kim KJ, Jahng TA, et al. Minimally Invasive Robotic Versus Open Fluoroscopic-guided Spinal Instrumented Fusions: A Randomized Controlled Trial. Spine (Phila Pa 1976) 2017;42:353-8.

19. Tian W, Fan M, Liu Y. Pedicle screw insertion in spine: a randomized controlled study for robot-assisted spinal surgery. Available online: https://easychair.org/ publications/paper/GsWT

20. Roser F, Tatagiba M, Maier G. Spinal robotics: current applications and future perspectives. Neurosurgery 2013;72:12-8.

21. Ringel F, Stüer C, Reinke A, et al. Accuracy of robotassisted placement of lumbar and sacral pedicle screws: a prospective randomized comparison to conventional freehand screw implantation. Spine (Phila Pa 1976) 2012;37:E496-501.

22. Kim HJ, Jung WI, Chang BS, et al. A prospective, randomized, controlled trial of robot-assisted vs freehand pedicle screw fixation in spine surgery. Int J Med Robot 2017. doi: http://dx.doi.org/10.1002/rcs.1779.

23. Kim HJ, Lee SH, Chang BS, et al. Monitoring the quality of robot-assisted pedicle screw fixation in the lumbar spine by using a cumulative summation test. Spine (Phila $\mathrm{Pa}$ 1976) 2015;40:87-94.

24. Han X, Tian W, Liu Y, et al. Safety and accuracy of robotassisted versus fluoroscopy-assisted pedicle screw insertion in thoracolumbar spinal surgery: a prospective randomized controlled trial. J Neurosurg Spine 2019. doi: http:// dx.doi.org/10.3171/2018.10.SPINE18487.

25. Gertzbein SD, Robbins SE. Accuracy of pedicular screw placement in vivo. Spine 1990;15:11-4.

26. Schizas C, Michel J, Kosmopoulos V, et al. Computer tomography assessment of pedicle screw insertion in percutaneous posterior transpedicular stabilization. Eur Spine J 2007;16:613-7.

27. Wolf A, Shoham M, Michael S. Feasibility study of a 
mini, bone-attached, robotic system for spinal operations: analysis and experiments. Spine (Phila Pa 1976) 2004;29:220-8.

28. Pechlivanis I, Kiriyanthan G, Engelhardt M. Percutaneous placement of pedicle screws in the lumbar spine using a bone mounted miniature robotic system: first experiences and accuracy of screw placement. Spine (Phila Pa 1976) 2009;34:392-8.

29. Barzilay Y, Liebergall M, Fridlander A. Miniature robotic guidance for spine surgery-introduction of a novel system and analysis of challenges encountered during the clinical development phase at two spine centres. Int J Med Robot 2006;2:146-53.

30. Wang JQ, Wang Y, Feng Y, et al. Percutaneous Sacroiliac Screw Placement: A Prospective Randomized Comparison of Robot-assisted Navigation Procedures with a Conventional Technique. Chin Med J (Engl)

Cite this article as: Peng YN, Tsai LC, Hsu HC, Kao CH. Accuracy of robot-assisted versus conventional freehand pedicle screw placement in spine surgery: a systematic review and meta-analysis of randomized controlled trials. Ann Transl Med 2020;8(13):824. doi: 10.21037/atm-20-1106
2017;130:2527-34.

31. Tian W. Robot-Assisted Posterior C1-2 Transarticular Screw Fixation for Atlantoaxial Instability: A Case Report. Spine (Phila Pa 1976) 2016;41:B2-B5.

32. Tian W, Wang H, Liu YJ. Robot-assisted Anterior Odontoid Screw Fixation: A Case Report. Orthop Surg 2016;8:400-4.

33. Mechlenburg I, Henrik D, Kjeld Søballe. Radiation exposure to the orthopaedic surgeon during periacetabular osteotomy. Int Orthop 2009;33:1747-51.

34. Malik AT, Rai HH, Lakdawala RH, et al. Does surgeon experience influence the amount of radiation exposure during orthopedic procedures? A systematic review. Orthop Rev (Pavia) 2019;11:7667.

35. Menger RP, Savardekar AR, Farokhi F, et al. A CostEffectiveness Analysis of the Integration of Robotic Spine Technology in Spine Surgery. Neurospine 2018;15:216-24. 